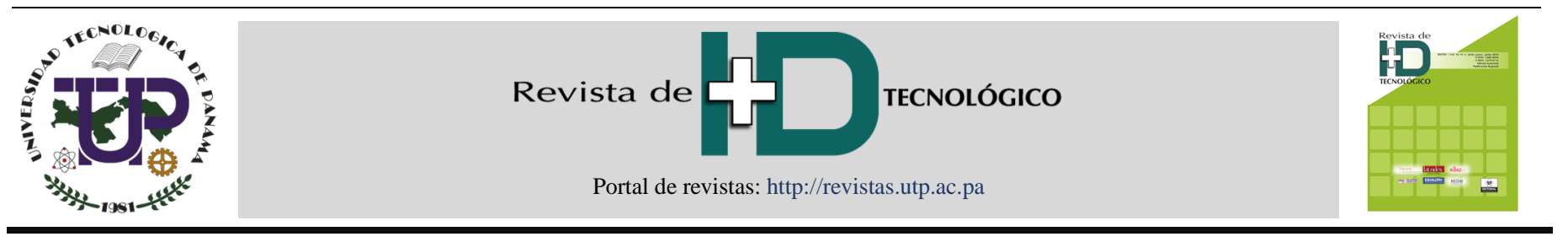

\title{
Optimización en el dimensionamiento de un sistema industrial de gas licuado de petróleo
}

\section{Optimization in the sizing of an industrial system of liquefied petroleum gas}

\author{
Diego Venegas Vásconez ${ }^{1 *}$, Cesar Ayabaca Sarria ${ }^{2}$, Santiago Celi Ortega ${ }^{3}$, Juan Rocha Hoyos ${ }^{4}$, Euro Mena Mena ${ }^{5}$ \\ ${ }^{1}$ Facultad de Ingeniería, Universidad de Concepción, Chile \\ ${ }^{2}$ Departamento de Ingeniería Mecánica, Escuela Politécnica Nacional, Ecuador \\ ${ }^{3,4}$ Facultad de Arquitectura e Ingeniería, Universidad Internacional SEK, Ecuador \\ ${ }^{5}$ Departamento de Energía y Mecánica, Universidad de las Fuerzas Armadas, Ecuador \\ *Autor de correspondencia: diegofvenegas@udec.cl
}

\begin{abstract}
RESUMEN-Son muchos los equipos industriales que requieren del gas licuado de petróleo (GLP) como combustible para su funcionamiento y para que llegue a estos equipos, se necesita entre otros de sistemas de almacenamiento (tanques) y de transporte (tuberías); su instalación debe estar acorde a principios de seguridad (distancias mínimas a terceros, materiales de tuberías) establecidos en la norma técnica que impone la autoridad competente y su dimensionamiento debe responder a criterios ingenieriles de transferencia de calor, termodinámica y mecánica de fluidos.

En el presente trabajo se optimiza la ubicación de los recipientes estacionarios y el diámetro de tubería necesarios para abastecer de combustible a un sistema industrial de GLP en la ciudad de Panamá, que cuenta con un horno de $600 \mathrm{~kW}$ y 6 quemadores industriales de $60 \mathrm{~kW}$ c/u. En el almacenamiento se compara el área superficial requerida por una batería de tanques estacionarios (250, 500, 1000 y $2000 \mathrm{Gl})$ y de acuerdo a las distancias de seguridad requeridas se minimiza esta área. En el dimensionamiento de tubería se establecen dos tramos de media presión (tubería entre el regulador de primera y segunda etapa) y un tramo de baja presión (tubería después del regulador de segunda etapa) para los seis quemadores, y en cada caso se plantean tres criterios de cálculo y se selecciona aquella tubería que arroja como resultado el menor diámetro.
\end{abstract}

Palabras claves- Gas licuado de petróleo, optimización, tanques, tuberías, dimensionamiento.

ABSTRACT - Many industrial equipment requires liquefied petroleum gas (LPG) as fuel for its operation. To reach these equipment, you need among others storage systems (tanks) and transport (pipelines); its installation must be in accordance with safety principles (minimum distances to third parties, pipe materials) established in the Technical Standard imposed by the competent authority and its dimensioning must respond to engineering criteria of heat transfer, thermodynamics and fluid mechanics.

In the present work, we optimize location of the stationary vessels and the diameter of the pipes necessary to supply fuel to an LPG industrial system in Panama City, which has a $600 \mathrm{~kW}$ furnace and 6 industrial $60 \mathrm{~kW}$ burners are optimized. /or. In the storage the surface area required by a battery of stationary tanks $(250,500,1000$ and $2000 \mathrm{Gl})$ is compared and according to the required safety distances this area is minimized. In the sizing of the pipe, two sections of medium pressure (pipe between the first and second stage regulator) and a low pressure section (pipe after the second stage regulator) are established for the 6 burners, and in each case three calculation criteria and select the pipeline that results in the smallest diameter.

Keywords-Liquefied petroleum gas, optimization, tanks, pipelines, sizing.

\section{Introducción}

Los usos y aplicaciones del GLP van desde consumos residenciales [1], comerciales [2] hasta industriales [3] en la generación de energía eléctrica, calentamiento de hornos y calderas, entre otros, y se lo escoge entre otros combustibles ya que: se pueden satisfacer varias necesidades energéticas con una sola fuente de combustible [4], tiene alto poder calorífico comparado con otras fuentes de energía [5], es limpio en término de emisiones de contaminantes a la atmósfera [6], no es tóxico para el ser humano [7], y es muy seguro si se lo maneja técnicamente [8].

Al ser un combustible muy explosivo e inflamable [9], su manejo genera riesgos hacia las personas y edificaciones a su alrededor [10], por lo que se han establecido criterios mínimos de seguridad en Normas Técnicas tales como la NFPA 54 [11] y NFPA 58 [12], que establecen entre otras cosas distancias mínimas de seguridad hacia terceros, formas de ubicar dichos

Citación: D. Vanegas, C. Ayabaca, S. Celi, J. Rocha y E. Mena, "Optimización en el dimensionamiento de un sistema industrial de gas licuado de petróleo", Revista de I+D Tecnológico, vol. 14, n. ${ }^{\circ} 1$, pp. 41-48, Jun. 2018.

Tipo de artículo: Original. Recibido: 22 de noviembre de 2017. Recibido con correcciones: 19 de enero de 2018 . Aceptado: 11 de abril de 2018.

Copyright: 2018 D. Vanegas, C. Ayabaca, S. Celi, J. Rocha y E. Mena. This is an open access article under the CC BY-NC-SA 4.0 license

(https://creativecommons.org/licenses/by-nc-sa/4.0/). 
recipientes y protecciones con el fin de reducir la posibilidad de eventos catastróficos en su entorno [13].

En instalaciones industriales, donde los requerimientos del combustible son elevados, es aconsejable la instalación de tanques estacionarios, sin embargo, los profesionales que se dedican a instalar este tipo de sistemas se encuentran con dificultades para encontrar los espacios más adecuados para ubicarlos dadas las limitaciones de espacio físico en las edificaciones, esto hace que se haga un análisis exhaustivo con el fin de encontrar la mejor opción que no comprometa la seguridad tanto física como humana.

En este almacenamiento el combustible se encuentra en fase gaseosa, pero para el consumo, salvo en aplicaciones específicas, el combustible debe estar en fase vapor. El cambio de fase se produce al interior de los recipientes como un fenómeno de vaporización natural que se da por la transferencia de calor desde el medio ambiente hacia este hidrocarburo a través de sus paredes [14], y está directamente relacionada con:

- La superficie delimitada por el GLP que está en estado líquido (zona mojada) [15], mientras mayor sea esta zona mojada, mayor será la capacidad de vaporización.

- A mayor tamaño del recipiente, mayor será la zona mojada y por lo tanto tendrá mayor capacidad de vaporización.

- A mayor temperatura ambiente habrá mayor transferencia de calor hacia el combustible, por tanto, la capacidad de vaporización va a aumentar.

En la ecuación 1 se indica la capacidad de vaporización natural de un recipiente de almacenamiento [16]:

$$
\mathrm{Q}=p \times S \times K \times \frac{\left(\mathrm{T}_{\mathrm{e}}-\mathrm{T}_{\mathrm{g}}\right)}{C L V}
$$

El líquido vaporizado llega a los equipos de consumo a través de sistemas de tuberías, cuyos materiales [17] y criterios de seguridad para su instalación [18] también se encuentran delimitados en la norma técnica. Estas tuberías deben como mínimo soportar la acción del combustible, y su dimensión (diámetro) debe ser el suficiente para permitir el paso del gas en la cantidad (caudal) y la presión necesaria en los equipos de consumo para su funcionamiento. Sin embargo se han detectado varias instalaciones cuyas tuberías han sido sobredimensionadas causando gastos innecesarios en materiales a los usuarios finales.

Este trabajo se presenta el dimensionamiento de un sistema industrial de GLP en la ciudad de Panamá, donde se plantean algunos criterios para optimizar la ubicación de recipientes de almacenamiento (área de ubicación), y diámetros mínimos de tubería, que garanticen el funcionamiento de los equipos de consumo. Las unidades utilizadas para los cálculos son las aceptadas por el sistema internacional, sin embargo para la presentación de algunos resultados se utilizan unidades con las cuales se identifican comercialmente algunos productos necesarios en estos sistemas como la capacidad de almacenamiento de los recipientes (Gl) y el diámetro de tuberías (plg).

\section{Metodología}

Se va a proceder a optimizar el almacenamiento (área mínima ocupada por los tanques) y los diámetros óptimos de tuberías para conducción del fluido hasta los equipos de consumo. Para ello es necesario conocer la potencia total requerida en la instalación, la cual será igual a la sumatoria de la potencia requerida por cada equipo de consumo según se muestra en la ecuación 2.

$$
P_{\text {tot }}=\sum_{i=1}^{n} p_{i}
$$

Obtenida la potencia total instalada en el sistema, se puede entonces dimensionar los recipientes de almacenamiento del combustible y las tuberías de transporte de este hacia los equipos de consumo.

\subsection{Optimización en el almacenamiento (tanques)}

La potencia total instalada mostrada en la ecuación 2 se satisface desde los recipientes, en donde se produce la vaporización natural (ecuación 1). Para garantizar que el usuario no se quede sin combustible durante la operación de su sistema, se recomienda calcular la vaporización de los recipientes al 20\% de llenado [19], y esta debe ser mayor o igual que la potencia total instalada según se muestra en la ecuación 3.

$$
Q \geq P_{\text {tot }}
$$


La instalación de estos recipientes debe estar acorde a la norma técnica vigente, y en ella se establecen distancias de seguridad hacia terceros que puedan potenciar un incidente con fuego y se establecen distancias mínimas entre recipientes para facilitar tareas de inspección y mantenimiento. En la ecuación 4 se muestra el área mínima requerida para la ubicación de recipientes considerando la distancia mínima desde sus paredes hacia terceros [20][21], y en la ecuación 5 se calcula el área mínima para delimitar los recipientes considerando la distancia recomendada en la Norma para separación entre ellos.

$$
\begin{gathered}
A_{\text {Seg }}=\left(\left(n \times D_{r}\right)+(n-1) d_{\text {Seg }}+2 d_{\text {Seg }}\right) \times\left(L_{r}+2 d_{\text {Seg }}\right) \\
A_{\text {Min }}=\left(\left(n \times D_{r}\right)+(n+1) d_{\text {min }}\right) \times\left(L_{r}+2 d_{\text {min }}\right)
\end{gathered}
$$

Aquel grupo de recipientes que tenga la menor área de seguridad $\left(\mathrm{A}_{\mathrm{Seg}}\right)$ y $\left(\mathrm{A}_{\mathrm{Min}}\right)$ será el recomendado para la instalación.

Otro criterio para tomar en cuenta es la autonomía del sistema, definida como el tiempo transcurrido entre cada abastecimiento de combustible, con esta medida se coordina con la comercializadora para el despacho del gas (ecuación 6) [14][22].

$$
\mathrm{A} u t=\frac{\left(\mathrm{n} \times{\mathrm{C} a p_{\text {real }}} \times \mathrm{C}_{\mathrm{p}}\right)}{P_{\text {ins }} \times h}
$$

\subsection{Optimización en el transporte (diámetro mínimo de tuberías)}

Para determinar los diámetros óptimos hay varios criterios que se aplican según la tubería sea instalada en servicio de media presión (entre regulador de primera y segunda etapa) o baja presión (después del regulador de segunda etapa) [23].

Los criterios seleccionados para dimensionar las tuberías en media presión son:

Renouard [24][25][26].

$$
p_{1}^{2}-p_{2}^{2}=4810 \times L \times d \times Q_{s}^{1,82} \times D^{-4,82}
$$

Spitzglass [27]

$$
Q_{s}=0,02094\left(\frac{D^{5}}{1+\frac{0,09144}{D}+1,1811 D}\right)^{0,5}\left(\frac{p_{1}^{2}-p_{2}^{2}}{d \times L}\right)^{0,5}
$$

Fristche [28]

$Q_{s}=94,2565 \frac{T_{s}}{P_{s}} D^{2,6811}\left(\frac{\left(p_{1}^{2}-p_{2}^{2}\right)-E}{L \times T \times d^{0,858}}\right)^{0,5}$

Para baja presión los criterios utilizados son:

\section{Renouard [24]}

$$
Q_{S}=8,641 \times D^{\frac{4,82}{1,82}} \times\left(\frac{p_{1}-p_{2}}{L \times d^{0,82}}\right)^{\frac{1}{1,82}}
$$

Spitzglass [27]

$$
Q_{s}=9,5 \times\left(\frac{\left(p_{1}-p_{2}\right) \times D^{5}}{L \times d \times\left(1+\frac{0,09144}{D}+1,1811 \times D\right)}\right)^{0,5}
$$

Pole [28]

$$
Q_{s}=C \times\left(\frac{\left(p_{1}-p_{2}\right) \times D^{5}}{L \times d}\right)^{0,5}
$$

Las consideraciones para determinar el diámetro óptimo de tubería son las siguientes:

- Se debe definir si la sección de tubería se encuentra entre la primera y segunda etapa de regulación (media presión), o está después del regulador de segunda etapa (baja presión). 
- La ruta por donde se colocará la tubería será la más corta posible entre el almacenamiento y los puntos de consumo, por tanto, el trazado de tubería será lo más recto posible, evitando en lo posible quiebres y cambios de sección abruptos.

- Se dividirá el trazado de tubería en secciones según la ubicación de equipos de consumo intermedios.

- Se requiere establecer la longitud de cada una de las secciones, para lo cual se puede aplicar el concepto de longitud equivalente, cuyo valor incluye una longitud de tubería asociada a las pérdidas que ocurren en las paredes de las tuberías por fricción y en cada uno de los accesorios. Se puede asumir una longitud equivalente de tubería según la ecuación 13 [29]:

$$
L_{e q}=L \times 1,2
$$

Encontrados los valores del diámetro que garantiza la conducción del fluido con cada uno de los criterios planteados en las ecuaciones (Ec. 2) hasta la (Ec. 7) se debe ajustar dicho valor a los diámetros de tuberías comercialmente disponibles, y entre los resultados se seleccionará el menor.

\section{Presentación y análisis de resultados}

La tabla 1 muestra la potencia total instalada del sistema, aplicando la (Ec. 8).

Tabla 1. Equipos de consumo de GLP

\begin{tabular}{|c|c|c|c|}
\hline Equipos & Cantidad & $\begin{array}{c}\mathbf{P}_{\mathbf{i}} \\
\mathbf{k W}\end{array}$ & $\begin{array}{c}\mathbf{P}_{\text {tot }} \\
\mathbf{k W}\end{array}$ \\
\hline Quemador & 6 & 60 & 360 \\
\hline Horno & 1 & 600 & 600 \\
\hline Total & $\mathbf{7}$ & & $\mathbf{9 6 0}$ \\
\hline
\end{tabular}

Esta potencia instalada $(960 \mathrm{~kW})$ debe satisfacerse desde los recipientes y transportarse a través de las tuberías.

\subsection{Optimización en el almacenamiento (recipientes)}

La tabla 2 muestra la capacidad natural de vaporización de recipientes estacionarios para almacenamiento de GLP (ecuación 1), aplicando lo siguiente: $\mathrm{p}=0,336$ [16], $\mathrm{K}=0,016\left[\mathrm{~kW} / \mathrm{m}^{2}{ }^{\circ} \mathrm{C}\right][16], \mathrm{T}_{\mathrm{e}}$ $=22,5\left[{ }^{\circ} \mathrm{C}\right][30], \mathrm{T}_{\mathrm{g}}=-26\left[{ }^{\circ} \mathrm{C}\right][16], \mathrm{CLV}=0,11$ $[\mathrm{kWh} / \mathrm{Kg}][16]$.

Tabla 2. Capacidad de vaporización de recipientes estacionarios

\begin{tabular}{|c|c|c|c|c|c|c|c|c|c|c|}
\hline Capreal & Diámetro & Longitud & $\mathbf{Q}$ & $\mathbf{n}$ & Aut & $\mathbf{D}_{\text {Seg }}$ & $\mathbf{A}_{\text {seg }}$ & $\mathbf{D}_{\text {Min }}$ & $\mathbf{A}_{\text {Min }}$ & Aut \\
\hline Gl [31] & $\mathbf{m ~ [ 3 1 ]}$ & $\mathbf{m ~ [ 3 1 ]}$ & $\mathbf{K g} / \mathbf{h} \mathbf{( k W )}$ & & $\mathbf{d i ́ a}$ & $\mathbf{m}[12]$ & $\mathbf{m}^{\mathbf{2}}$ & $\mathbf{m}[12]$ & $\mathbf{m}^{\mathbf{2}}$ & $\mathbf{d i ́ a}$ \\
\hline 250 & 0,80 & 2,20 & $13,1(182)$ & 6 & 23 & 7,6 & 1009 & 1 & 49,56 & 23 \\
\hline 500 & 0,95 & 2,99 & $21,14(293,7)$ & 4 & 19 & 7,6 & 760,3 & 1 & 43,91 & 19 \\
\hline 1000 & 1,04 & 4,85 & $37,54(521,6)$ & 2 & 11 & 7,6 & 498,8 & 1 & 34,8 & 11 \\
\hline 2000 & 1,18 & 7,24 & $63,59(883,4)$ & 2 & 13 & 15 & 1764 & 1,5 & 70,25 & 13 \\
\hline
\end{tabular}

La tabla 2 muestra además la cantidad de recipientes necesarios para garantizar el abastecimiento de GLP, (ecuación 3) cuando estos se encuentren al 20\% de llenado, el área mínima de seguridad (ecuación 4) requerida para cumplir con las distancias según el volumen instalado, el área mínima de circulación (ecuación 5) establecida como aquella área en la cual se podrían hacer trabajos de mantenimiento sin dificultades y la autonomía del sistema, es decir los días que se tiene combustible en los recipientes hasta la siguiente recarga (ecuación 6).
De acuerdo a los resultados obtenidos con respecto a la instalación de grupo de recipientes se puede decir lo siguiente:

- La opción de 2 recipientes de 1000 Gl. c/u ofrece la mejor alternativa en los criterios de menor área de seguridad $\left(498,8 \mathrm{~m}^{2}\right)$ y menor área mínima $\left(34,8 \mathrm{~m}^{2}\right)$.

- La opción de 6 recipientes de 250 Gl c/u es la mejor alternativa si el criterio de selección es la autonomía.

- La opción de 2 recipientes de 2000 Gl. c/u ofrece la alternativa menos favorable en los criterios de menor 
área de seguridad $\left(1764 \mathrm{~m}^{2}\right)$ y menor área mínima $\left(70,25 \mathrm{~m}^{2}\right)$.

- Los grupos de 6 recipientes de 250 Gl, 4 recipientes de $500 \mathrm{Gl}$ y 2 recipientes de $1000 \mathrm{Gl}$ tienen las mismas distancias de seguridad hacia terceros $(7,6 \mathrm{~m})$ y distancia mínima entre recipientes $(1 \mathrm{~m})$.

Pueden buscarse configuraciones donde se combinen recipientes de diferentes capacidades, sin embargo, esto no es aconsejable ya que el vaciado de estos no va a ser homogéneo, y eso traerá problemas de caída de presión en el sistema.

La figura 1 muestra la disposición final del grupo de recipientes que optimizan el sistema $(2$ recipientes de $1000 \mathrm{Gl}$. c/u. y de las distancias mínimas que se deben respetar con respecto a la ubicación entre estos recipientes, la limitación para circulación de personal de mantenimiento e inspección y la distancia de seguridad desde sus paredes con respecto a terceros que pueden ser elementos que incrementen el riesgo del almacenamiento del combustible.

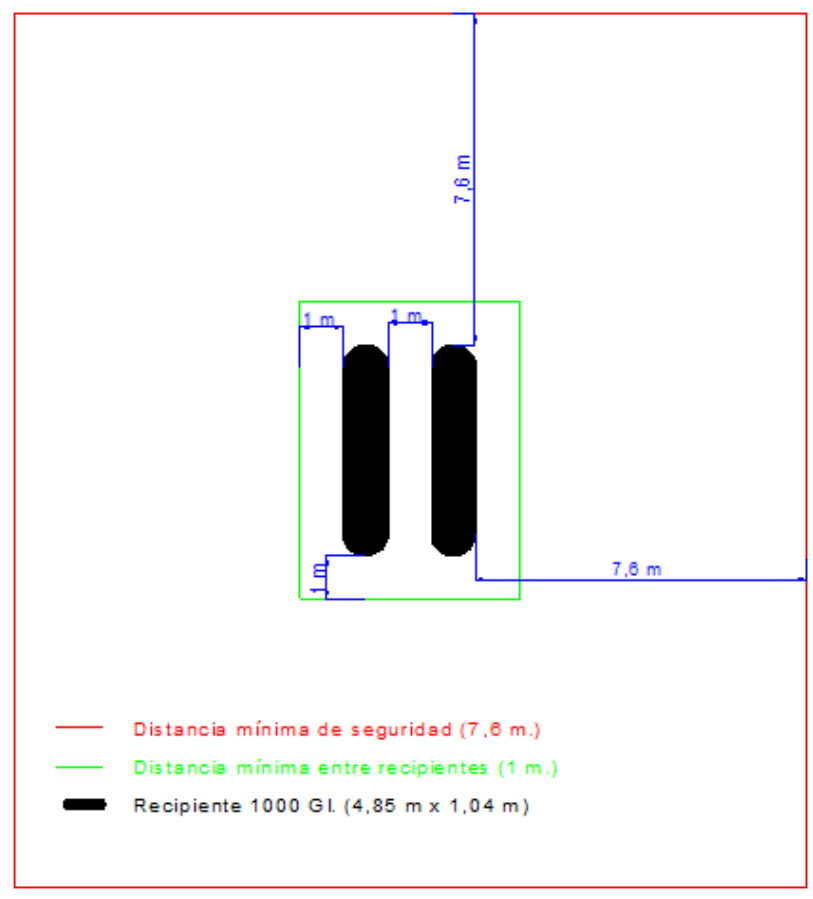

Figura 1. Mínima distancia de seguridad, mínima distancia entre recipientes y menor área de seguridad.

\subsection{Optimización en el diámetro de tuberías}

Aplicados los criterios para determinación del diámetro óptimo de tubería, y según la ubicación de reguladores de presión se puede determinar lo siguiente:

\section{a) Media presión:}

Para determinar los diámetros mínimos en media presión se utilizan las ecuaciones 7,8 y 9 además de las siguientes constantes:

Tramo 1: Qs $=960[\mathrm{~kW}], \mathrm{L}=38[\mathrm{~m}], \mathrm{Le}=45,6$ [m], p1 = 1,2 [Bar]; p2 = 1,08 [Bar] (10\% de caída de la presión manométrica [32]), $\mathrm{d}=1,5[12], \mathrm{E}=0\left[\mathrm{~Pa}^{2}\right]$ [12].

Tramo 2: Qs $=360[\mathrm{~kW}], \mathrm{L}=40[\mathrm{~m}], \mathrm{Le}=48[\mathrm{~m}]$, p1 = 1,2 [Bar]; p2 = 1,08 [Bar] $(10 \%$ de caída de la presión manométrica [32]), $\mathrm{d}=1,5[12], \mathrm{E}=0\left[\mathrm{~Pa}^{2}\right]$ [12].

La tabla 3 muestra los resultados de los diámetros obtenidos de acuerdo a los criterios planteados, para cada uno de los tramos, y como se tienen comercialmente diámetros definidos, aquellos resultados intemedios deben ser ajustados a los diámetros comerciales inmediatamente superiores.

Tabla 3. Diámetros de tubería (media presión)

\begin{tabular}{|c|c|c|c|c|c|c|}
\hline Tramo & \multicolumn{2}{|c|}{ Renouard } & \multicolumn{2}{c|}{ Spitzglass } & \multicolumn{2}{c|}{ Fritzche } \\
\hline & Calc & Com & Calc & Com & Calc & Com \\
\hline & plg & plg & plg & plg & plg & plg \\
\hline $\mathbf{1}$ & 0,921 & 1 & 1,04 & $1 \frac{1 / 4}{4}$ & 0,925 & 1 \\
\hline $\mathbf{2}$ & 0,660 & $3 / 4$ & 0,765 & 1 & 0,666 & $3 / 4$ \\
\hline
\end{tabular}

En la figura 2 se muestra la comparación de los diámetros obtenidos y el ajuste a realizar al diámetro comercialmente superior.

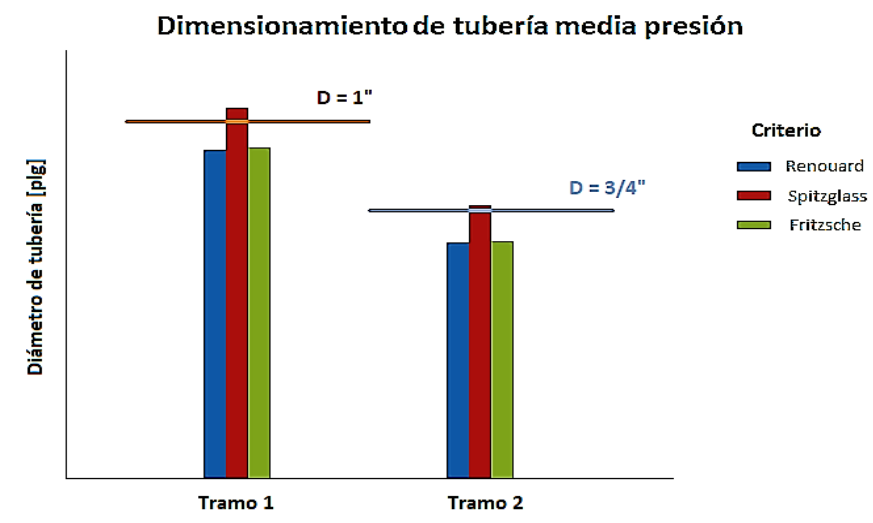

Figura 2. Diámetros de tuberías calculados vs. diámetros comerciales disponibles para media presión. 
Para los tramos $1(960 \mathrm{~kW})$ y $2(360 \mathrm{~kW})$, los criterios de Renouard y Flitzche entregan resultados de diámetros bajo 1" y 3/4" respectivamente. El criterio de Spitzglass es más conservador y arroja unos resultados de $11 / 4$ " y 1 " respectivamente, y si escogeríamos este criterio estaríamos sobredimensionando el sistema.

\section{b) Baja presión}

Se utilizan las ecuaciones 10, 11 y 12 con las siguientes constantes para el cálculo en baja presión, con las siguientes constantes:

$\mathrm{Qs}=180[\mathrm{~kW}], \mathrm{L}=16[\mathrm{~m}], \mathrm{Le}=19,2[\mathrm{~m}], \mathrm{p}_{1}-\mathrm{p}_{2}=$ 150 [Pa] [33], $\mathrm{d}=1,5$ [12], $\mathrm{C}=4,635[27]$.

La tabla 4 muestra los resultados de los diámetros obtenidos para baja presión de acuerdo a los criterios planteados.

Tabla 5. Diámetros de tubería (baja presión)

\begin{tabular}{|c|c|c|c|c|c|}
\hline \multicolumn{2}{|c|}{ Renouard } & \multicolumn{2}{c|}{ Spitzglass } & \multicolumn{2}{c|}{ Fritzche } \\
\hline Calc & Com & Calc & Com & Calc & Com \\
\hline plg & plg & plg & plg & plg & plg \\
\hline 0,976 & 1 & 1,08 & $1 \frac{1 / 4}{1,072}$ & 1 \\
\hline
\end{tabular}

En la figura 3 se muestran los diámetros calculados que han sido ajustados al diámetro comercial inmediatamente superior.

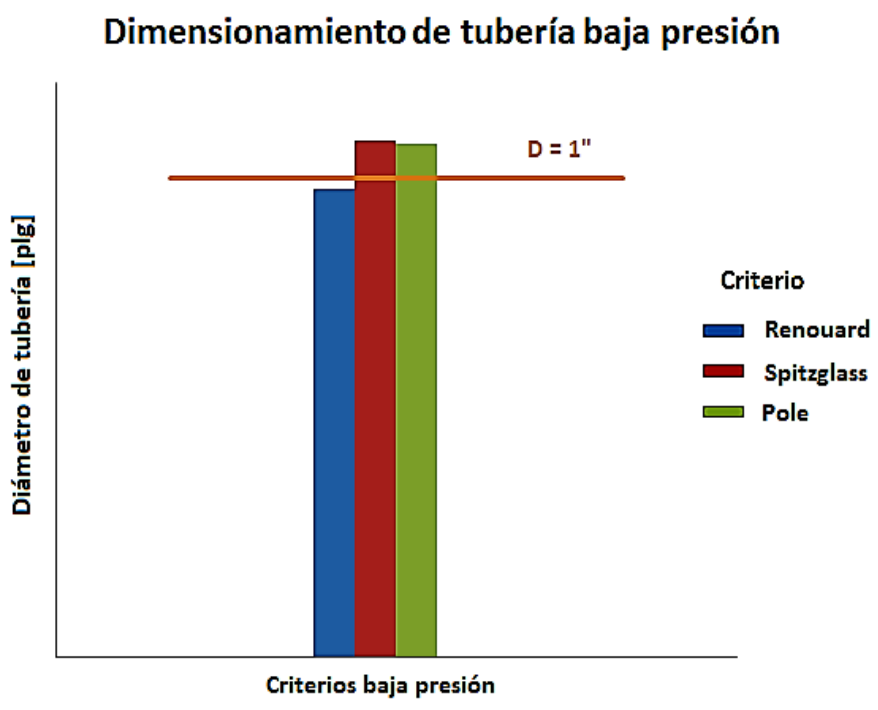

Figura 3. Diámetros de tuberías calculados vs. diámetros comerciales disponibles para baja presión.
El criterio de Renouard entrega un resultado de diámetro bajo 1". Los criterios de Spitzglass y Pole son más conservadores y su resultado debe aproximarse a un diámetro comercial de $1 \frac{1 / 4}{4}$, esto indica que si se escogen los criterios o de Spitzglass Pole, se estará sobredimensionando el sistema, se deberá comprar una tubería de diámetro mayor, y por ende se encarece el proyecto.

La disposición final de la tubería según los tramos y los criterios escogidos se puede observar en la figura 4 .

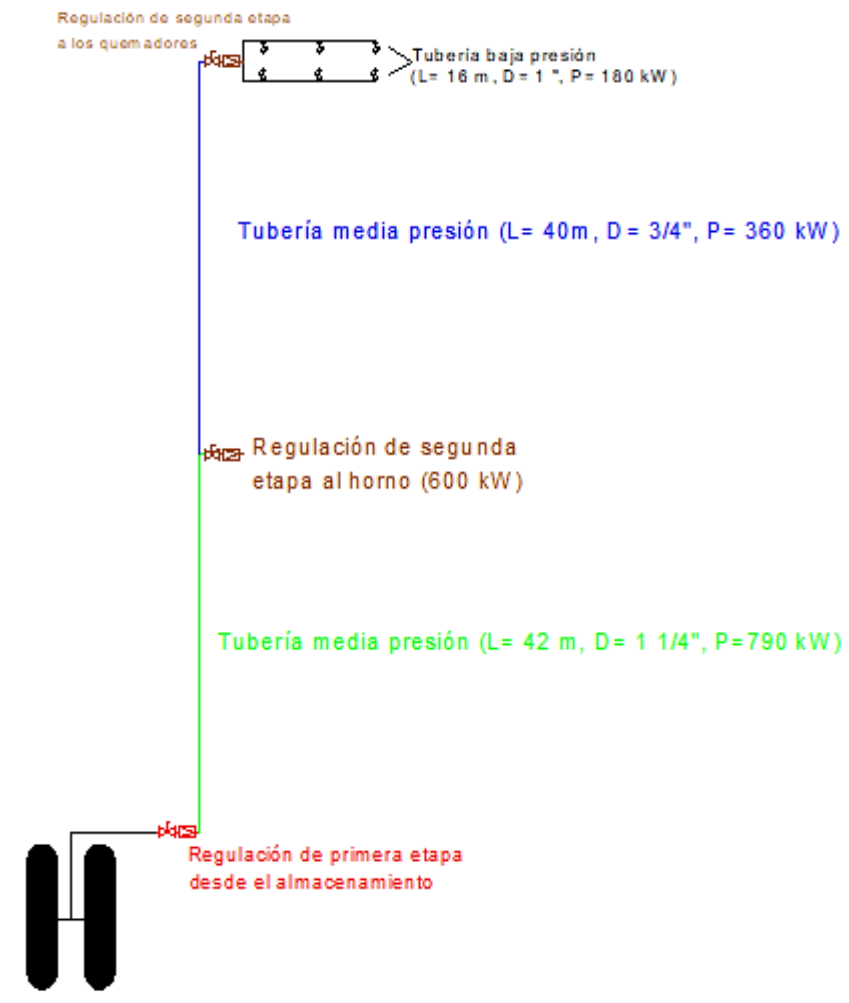

Figura 4. Tramos de tuberías de distribución.

\section{Conclusiones}

- Dimensionar adecuadamente un sistema de conducción de GLP garantiza satisfacer el funcionamiento adecuado de los equipos de consumo según su requerimiento de caudal y presión.

- Al respetar las distancias de seguridad establecidas en las normativas desde los recipientes hacia terceros se disminuye el riesgo generado por el almacenamiento del combustible.

- Un óptimo dimensionamiento de un sistema va a generar ahorros significativos en los usuarios, al no tener que invertir en tuberías y accesorios de diámetros mayores. 
- Un óptimo dimensionamiento parte de los datos correctos de los usuarios sobre las potencias de los equipos de consumo que va a disponer en su sistema y las horas proyectadas de consumo.

- No siempre es posible encontrar espacio disponible en una planta industrial para colocar los recipientes, por lo que se hace indispensable encontrar mecanismos para poder optimizar su ubicación.

- Si se prevé realizar futuras ampliaciones con nuevos equipos, se puede optar por dimensionar tuberías con criterios más conservadores, sobredimensionando inicialmente al sistema.

\section{Referencias}

[1] D. Kimemia and H. Annegarn, "Domestic LPG interventions in South Africa: Challenges and lessons," Energy Policy, vol. 93, pp. 150-156, 2016.

[2] L. Raslavi, S. Mockus, N. Ker, and M. Starevi, "Liquefied petroleum gas (LPG) as a medium-term option in the transition to sustainable fuels and transport," Renew. Sustain. Energy Rev., vol. 32, pp. 513-525, 2014.

[3] K. J. Morganti, T. Mun, M. J. Brear, Y. Yang, and F. L. Dryer, "The Research and Motor octane numbers of Liquefied Petroleum Gas (LPG),” Fuel, vol. 108, pp. 797-811, 2013.

[4] R. Kities, P. Mulder, and P. Rietveld, "Energy poverty reduction by fuel switching. Impact evaluation of the LPG conversion program in Indonesia," Energy Policy, vol. 66, pp. 436-449, 2014.

[5] P. Boggavarapu, B. Ray, and R. V Ravikrishna, "Thermal Efficiency of LPG and PNG-fired burners : Experimental and numerical studies," Fuel, vol. 116, pp. 709-715, 2014.

[6] L. Raslavi, S. Mockus, N. Ker, and M. Starevi, "Liquefied petroleum gas (LPG) as a medium-term option in the transition to sustainable fuels and transport," Renew. Sustain. Energy Rev., vol. 32, pp. 513-525, 2014.

[7] F. Chica, F. Espinoza, and N. Rivera, "Gas licuado de petróleo como combustible alternativo para motores diesel con la finalidad de reducir la contaminación del aire," Ingenius Rev. Cienc. y Tecnol., vol. 4, pp. 73-81, 2010.

[8] D. Venegas Vásconez, S. Celi Ortega, J. Rocha Hoyos, C. Ayabaca Sarria, and E. Mena Mena, "Manejo inseguro del gas licuado de petróleo en Panamá," RIDTEC, vol. 13, no. 2, pp. 22-30, 2017.

[9] M. A. Johnsen and G. Nardini, "Manual de seguridad: Aspectos de inflamabilidad de los gases hidrocarburos," Naciones Unidas, 2009.

[10] D. Venegas, "Seguridad en la instalacion de gases industriales (GLP)," in II Congreso Internacional de Energía República Dominicana, 2016, p. 14.

[11] NFPA, NFPA 54 Código Nacional de Gas Combustible. National Fire Protection Association, 2012.

[12] NFPA, NFPA 58 Código del Gas Licuado de Petróleo Edición 2014. National Fire Protection Association, 2014.
[13] D. Venegas and Ó. Farías, "La BLEVE, un motivo para la seguridad en las instalaciones de GLP," in $13^{\circ}$ Congreso Iberoamericano de Ingeniería Mecánica CIBEM, 2017.

[14] D. Venegas and C. Ayabaca, Instalaciones de gas licuado de petróleo, First Edit. 2017.

[15] S. P. Kumar, B. V. S. S. S. Prasad, G. Venkatarathnam, K. Ramamurthi, and S. S. Murthy, "Influence of surface evaporation on stratification in liquid hydrogen tanks of different aspect ratios," Int. J. Hydrogen Energy, vol. 32, pp. 1954-1960, 2007.

[16] E. López Sopeña, "Manual de instalaciones de GLP," 2001. [Online].

Available: http://www.blancogas.com/estilo/normas/bg/09-glp-cepsa.pdf.

[17] D. Venegas, M. Melendrez, and M. Arrocha, "Materiales para instalaciones de Gas Licuado de Petróleo según National Fire Protection Association (NFPA)," in SAM CONAMET, 2016, pp. 545-546.

[18] D. Venegas et al., "Materiales recomendados por las Normas Internacionales para utilizar en una instalación de GLP," in Asociacion Española de Ingenieria Mecanica, 2016, pp. 599606.

[19] A. Hernández and A. García, "Especificaciones técnicas CONAIF-SEDIGAS para la certificación de instaladores de gas. Materias comunes Tipos A, B y C. Depósitos fijos de GLP," 2008.

[20] T. Abbasi and S. A. Abbasi, "The boiling liquid expanding vapour explosion (BLEVE): Mechanism, consequence assessment, management," J. Hazard. Mater., vol. 141, pp. 489-519, 2007.

[21] T. Abbasi and S. A. Abbasi, "The boiling liquid expanding vapour explosion (BLEVE) is fifty ... and lives on!,” J. Loss Prev. Process Ind., vol. 21, no. 4, pp. 485-487, 2008.

[22] V. Salazar, G. Martinez, and J. Abad, "Modelo para la determinación del tamaño óptimo de un tanque estacionario de almacenamiento de GLP aplicado al negocio de comercialización al granel," Repos. la Esc. Super. Politécnica del Litoral, 2016.

[23] Fisher Emerson, Guía para el comprador de Equipos Equipos de Gas LP - 31. 2011.

[24] E. B. Brucart, Natural Gas. Barcelona, 1987.

[25] J. A. Andrés, R. Pomatta, S. A. Lastra, and M. . Gándara, Gaseous Fuels Installations. Madrid, 1997.

[26] J. L. . Becco, “Liquified Petroleum Gases,” Madrid, 1989.

[27] P. M. Coelho and C. Pinho, "Considerations about equations for steady state flow in natural gas pipelines," J. Brazilian Soc. Mech. Sci. Eng., vol. 29, no. 3, pp. 262-273, 2007.

[28] M. Mohitpour, H. Golshan, and A. Murray, Pipeline desing \& construction : A practical approach. 2000.

[29] A. Hernández and A. García, "Especificaciones técnicas CONAIF-SEDIGAS para la certificación de instaladores de gas. Materias comunes Tipos A, B y C. Cálculo de instalaciones receptoras," 2008.

[30] Climate-Data, "Clima: Panamá," 2017. [Online]. Available: https://es.climate-data.org/location/5885/.

[31] Trinity Industries de Mexico, "Tanques estacionarios para 
exportación," 2017. [Online]. Available: http://www.trinitymexico.com/files/tanques-estacionariospara-exportacion.pdf.

[32] RegO Products, "RegO Catálogo L-102-SV Equipo de Gas LP y Amoníaco Anhidro," 2011.

[33] Secretería de Energía y Combustibles Gobierno de Chile, Decreto $N^{o} 66$ Reglamento de Instalaciones Interiores $y$ Medidores de Gas. Ministerio de Economía, Fomento y Reconstrucción, 2007.

\section{Anexo}

\subsection{Nomenclatura}

$\mathrm{A}_{\mathrm{Min}}=$ Área mínima para circulación entre recipientes de GLP $\left[\mathrm{m}^{2}\right]$

$\mathrm{A}_{\text {Seg }}=$ Área de seguridad para recipientes de GLP $\left[\mathrm{m}^{2}\right]$

Aut $=$ Autonomía del sistema [día $]$

$\mathrm{C}=$ Constante en la Ecuación de Pole

Calc $=$ diámetro obtenido según fórmulas planteadas.

Cap $_{\text {real }}=$ Capacidad real del tanque $=0,65$ Capacidad total [Gl] (resta entre el máximo nivel de llenado (85\%) - el nivel mínimo de reserva de emergencia 20\%)

CLV = Calor latente de vaporización $[\mathrm{kWh} / \mathrm{Kg}]$

$\mathrm{Com}=$ diámetro comercial más cercano al calculado

$\mathrm{Cp}=$ capacidad calorífica del GLP [BTU/Gl]

$\mathrm{D}=$ diámetro interno de tubería $[\mathrm{m}]$

$\mathrm{d}=$ densidad relativa del gas

$\mathrm{D}_{\text {Min }}=$ Distancia mínima para circulación entre recipientes [m]

Dr = Diámetro del recipiente

$\mathrm{D}_{\mathrm{Seg}}=$ Distancia mínima de seguridad desde las paredes

de los recipientes hacia terceros [m]

$\mathrm{E}=$ Energía potencial térmica $\left[\mathrm{Pa}^{2}\right]$

$\mathrm{h}=$ horas al día de funcionamiento de equipos [hr/día]

$\mathrm{K}=$ Coeficiente de transmisión de calor a través de las paredes del recipiente $\left[\mathrm{kW} / \mathrm{m}^{2}{ }^{\circ} \mathrm{C}\right]$

$\mathrm{L}=$ longitud de tubería $[\mathrm{m}]$

$\mathrm{L}_{\mathrm{eq}}=$ longitud equivalente $[\mathrm{m}]$,

$\mathrm{Lr}=$ Longitud del recipiente

$\mathrm{n}=$ Número de recipientes instalados

$\mathrm{p}=$ Porcentaje de superficie mojada del depósito

$\mathrm{p}_{1}=$ presión absoluta de entrada a la tubería $[\mathrm{Pa}]$

$\mathrm{p}_{2}=$ presión absoluta de salida en la tubería $[\mathrm{Pa}]$

$\mathrm{P}_{\mathrm{i}}=$ Potencia individual del equipo de consumo [kW]

$\mathrm{P}_{\text {ins }}=$ Potencia instalada [BTU/hr]

$\mathrm{P}_{\mathrm{s}}=$ presión estándar, $1,013 \times 10^{5} \mathrm{~Pa}$

$\mathrm{P}_{\text {tot }}=$ Potencia total instalada del sistema $[\mathrm{kW}]$

$\mathrm{Q}=$ Capacidad de vaporización natural $[\mathrm{kW}]$

$\mathrm{Q}_{\mathrm{s}}=$ flujo volumétrico a condiciones estándar $\left[\mathrm{m}^{3} / \mathrm{s}\right]$

$\mathrm{S}=$ Superficie del recipiente [m2]
$\mathrm{Te}=$ Temperatura exterior mínima $\left[{ }^{\circ} \mathrm{C}\right]$

$\mathrm{Tg}=$ Temperatura de equilibrio líquido gas $\left[{ }^{\circ} \mathrm{C}\right]$

$\mathrm{T}_{\mathrm{s}}=$ temperatura estándar $288,15 \mathrm{~K}$ 